in vivo $33: 1893-1899$ (2019)

doi:10.21873/invivo.11683

\title{
Excessive Expression of Microglia/Macrophage and Proinflammatory Mediators in Olfactory Bulb and Olfactory Dysfunction After Stroke
}

\author{
CHIEN-FU YEH ${ }^{1,2,3}$, TUNG-YUEH CHUANG ${ }^{4}$, MING-YING LAN ${ }^{1,2}$, \\ YU-CHING CHIN ${ }^{5}$, WEI-HSIN WANG ${ }^{6}$ and YUNG-YANG LIN LI, $^{3,7,8,9}$ \\ ${ }^{1}$ Department of Otolaryngology-Head and Neck Surgery, Taipei Veterans General Hospital, Taipei, Taiwan, R.O.C.; \\ ${ }^{2}$ Department of Otorhinolaryngology, National Yang-Ming University, Taipei, Taiwan, R.O.C.; \\ ${ }^{3}$ Institute of Brain Science, National Yang-Ming University, Taipei, Taiwan, R.O.C.; \\ ${ }^{4}$ Department of Critical Care Medicine, Taipei Veterans General Hospital, Taipei, Taiwan, R.O.C.; \\ ${ }^{5}$ Department of Medical Education, Taipei Veterans General Hospital, Taipei, Taiwan, R.O.C.; \\ ${ }^{6}$ Department of Neurosurgery, Taipei Veterans General Hospital, \\ School of Medicine, National Yang-Ming University, Taipei, Taiwan, R.O.C.; \\ ${ }^{7}$ Institute of Physiology, National Yang-Ming University, Taipei, Taiwan, R.O.C.; \\ ${ }^{8}$ Institute of Clinical Medicine, National Yang-Ming University, Taipei, Taiwan, R.O.C.; \\ ${ }^{9}$ Department of Neurology, Neurological Institute, Taipei Veterans General Hospital, Taipei, Taiwan, R.O.C.
}

\begin{abstract}
Background/Aim: Olfactory dysfunction can be caused by stroke but the pathogenesis is still unclear. Previous studies have proved that olfactory dysfunction could be caused by microglia activation in the olfactory bulb and that middle cerebral artery occlusion (MCAO) may induce ipsilateral olfactory bulb microglia activation. This study aimed to explore the possible pathogenesis of ischemic stroke-induced olfactory dysfunction. Materials and Methods: We used a rat model of MCAO to simulate ischemic stroke. Olfactory function tests were performed using buried food test. The $M R N A$ expression of olfactory marker protein (OMP), microglia/macrophage activation, and proinflammatory mediators were measured using reverse transcription-quantitative polymerase chain reaction. Results: Following $M C A O$, rats had poorer olfactory performance. In the olfactory bulb of the rats, the $m R N A$ expression of OMP decreased and the $m R N A$
\end{abstract}

This article is freely accessible online.

Correspondence to: Yung-Yang Lin, Department of Critical Care Medicine, Taipei Veterans General Hospital, No. 201, ShiPai Rd., Taipei 112, Taiwan, R.O.C. Tel: +886 228757398, Fax: +886 228757579, e-mail: yylin@vghtpe.gov.tw

Key Words: Olfactory dysfunction, ischemic stroke, middle cerebral artery occlusion, rat, microglia, macrophage, proinflammatory mediator. expression of microglia/macrophage activation and proinflammatory mediators increased. Conclusion: Ischemic stroke causes microglia/macrophage activation and promotes neuroinflammation in the olfactory bulb, causing olfactory dysfunction.

Olfactory dysfunction includes anosmia, hyposmia and parosmia, and can significantly impair the quality of life of the patients. Olfactory dysfunction can be caused by upper respiratory tract infection, rhinosinusitis, head trauma and neurological diseases, such as stroke $(1,2)$. In the acute stage of stroke, $10 \%$ patients suffer from olfactory dysfunction (3). After stroke, $28.2 \%$ patients develop hyposmia and $15.4 \%$ patients have anosmia within one year (4). However, the pathogenesis of ischemic stroke-related olfactory dysfunction is still unclear.

Stroke can be classified into i) ischemic stroke and ii) hemorrhagic stroke with approximately $80 \%$ of strokes belonging to the former category (5). Ischemic stroke is caused by the interruption of blood supply to the brain, and the most common infarction site of ischemic stroke is the middle cerebral artery (MCA). The mechanism of cerebral damage of ischemic stroke comprises i) primary ischemia and ii) secondary neuroinflammation (6). During the process of neuroinflammation, the microglia, which are the brainresident macrophages, are activated and they release proinflammatory cytokines to cause brain tissue damage $(7,8)$. In addition to the ischemic brain, one study has revealed that the microglia in the ipsilateral olfactory bulb of mice are also 
activated after experimental stroke, at an earlier time compared to the ischemic brain (9).

Based on this, olfactory bulb microglia may play a role as modulators of neuroinflammation. In one animal study, Niemann-Pick disease type $\mathrm{C} 1$ mice presented with excessive microglia activation in their olfactory bulbs (10). In addition, they developed olfactory dysfunction and had decreased expression of olfactory marker protein (OMP), which is a marker of mature olfactory sensory neuron and is associated with odor discrimination $(10,11)$. The olfactory dysfunction improved when these mice were treated with the antiinflammatory agent Cyclosporin A, which inhibited microglia activation (10). Another study has shown that mice with LPSinduced rhinitis had olfactory bulb microglia activation and these mice also had olfactory dysfunction (12).

To date, there has been no study to explore the pathogenesis of ischemic stroke-related olfactory dysfunction. In the present study, we used a middle cerebral artery occlusion (MCAO) model in rats to investigate whether ischemic stroke can trigger microglia/macrophage activation as well as proinflammatory mediator production in the olfactory bulb, leading to olfactory dysfunction.

\section{Materials and Methods}

Animals. All animal experiments were approved by the Institutional Animal Care and Utilization Committee of Taipei Veterans General Hospital (IACUC certificate No. 2018-062). Ten weeks old male Wistar Kyoto (WKY) rats weighing 200-300 g were obtained from BioLASCO (Taipei, Taiwan). To avoid any influences of sex hormones, female rats were not used for any experiment. The rats lived in the animal room under a 12-hour light/dark cycle at a constant temperature $\left(25 \pm 2^{\circ} \mathrm{C}\right)$, and they had free access to food and water. The experimenters were blinded to the experimental conditions.

Focal cerebral ischemia with MCAO. Focal cerebral ischemia with MCAO was performed by surgical intervention, as we previously described (13). Briefly, rats were anesthetized with Xylazine $(8 \mathrm{mg} / \mathrm{kg})$ and Zoletil $(30 \mathrm{mg} / \mathrm{kg})$ by intraperitoneal injection. Bilateral common carotid arteries (CCAs) were identified and marked through a vertical incision in the midline of the neck. After retracting the temporal muscle, the right MCA was exposed through a craniotomy on the right temporal bone drilled by a cutting burr. The dura matter was cut, and the MCA was permanently occluded by suture ligation at the level of the olfactory tract. Subsequently, the bilateral CCAs were occluded temporarily for 90 minutes by a vessel clamp. Rats in the sham group were subjected to identical surgical procedures without MCAO. During MCAO, body temperature of the rats was maintained using a heat pad. Only rats undergoing successful MCAO were included for further treatment. Rats with massive bleeding during MCAO, rats that died during or after MCAO and rats with no significant infarction after MCAO were excluded. The mortality rate after MCAO was approximately $10 \%$.

Buried food test for olfaction evaluation. To evaluate the olfaction in rats, the buried food test was performed before MCAO and at 3 days after MCAO, as previously described with some modifications
(14). Briefly, the rats $(n=6)$ were fasted for 24 hours before the test. Then, the rats were individually put into a new cage with fresh bedding for 10 minutes for habituation. In the same time, a piece of chow was buried under the fresh bedding in the middle of a new cage (test cage) to a depth of $3 \mathrm{~cm}$. The rat was then placed in the corner of the test cage and was allowed to move freely to search for the buried food. The latency before discovering the food and beginning to eat was recorded. After the test, each rat was subjected to another trial with the food placed on top of the fresh bedding. This exposed food test was designed to ensure that the buried food test was based on olfaction and not on vision.

Quantification of infarct volume. At 3 days after MCAO, rats $(\mathrm{n}=4 /$ group $)$ were anesthetized with Xylazine $(8 \mathrm{mg} / \mathrm{kg})$ and Zoletil $(30 \mathrm{mg} / \mathrm{kg})$ by intraperitoneal injection and were subjected to transcardial perfusion with phosphate-buffered saline (PBS). The rats were decapitated and their brains along with olfactory bulbs were removed from the skull. To determine infarction size, 2-mmthick coronal sections were cut, stained with a 2\% 2,3,5-triphenyltetrazolium chloride (TTC; Sigma Chemical Co., St Louis, MO, USA) solution for 15 minutes at room temperature without light, and were then fixed with $4 \%$ paraformaldehyde. The non-infarcted region showed a red color, and the unstained area represented the infarcted region on TTC-stained sections. For brain edema correction, the infarcted region was calculated by subtracting the non-infarcted region in the ipsilateral hemisphere from the contralateral hemisphere. Infarct volumes were calculated as the sum of the infarcted regions on each brain slice using ImageJ software (The National Institutes of Health, Bethesda, MD, USA).

Measurement of the mRNA of microglia/macrophage activation and proinflammatory mediators. At 3 days after MCAO, total RNA was extracted from the olfactory bulb of the ipsilateral MCAO side and contralateral side ( $\mathrm{n}=4-5 /$ group), using E.Z.N.A. ${ }^{\circledR}$ HP Total RNA Kit (Omega Bio-tek Inc., Norcross, GA, USA). Total RNA of the olfactory bulb of the sham rats were also prepared as control. Subsequently, they were reverse-transcribed to cDNA with the assistance of a Thermo Scientific Revert Aid First Strand cDNA Synthesis Kit (Thermo Fisher Scientific Inc., Waltham, MA, USA). Reverse transcription quantitative polymerase chain reaction (PCR) was performed on an ABI QuantStudio 3 Real-Time PCR System (Applied Biosystems, Foster City, CA, USA) using SYBR green PCR Master Mix (Vazyme Biotech, Nanjing, PR China). The gene sequences of the primer pairs for microglia/macrophage marker and proinflammatory mediators are as follows (in pairs, forward, and reverse): i) Il-1 $\beta$ : 5'-CTCCACCTCAATGGACAGAACA-3' and 5'TTGCTTGTCTCTCCTTGTACAAAGC-3'; ii) Il-6: 5'-GCGATG ATGCACTGTCAGAAA-3' and 5'-TCCAGAAGACCAGAGCA GATTTT-3'; iii) Ccl2 (MCP-1): 5'-CTGTAGCATCCACGTG CTGTCT-3' and 5'-GCCGACTCATTGGGATCATC-3', iv) Omp: 5'-CCAGACCTCACCAACCTCATG-3' and 5'-CATGGCAT CGGAGTCTTCCT-3'; v) Aif1 (Iba1): 5'-CTGAAAGCCCAAC AGGAAGAGA-3' and 5'-CATCACTGCTGTACTTGGGATCAT3'; vi) Cd68: 5'-CACTTCGGGCCATGCTTCT-3' and 5'-CCAATG ATGAGAGGCAGCAA-3'; vii) Fcgr3a (CD16): 5'-TTGGAC GCAACAACATATCTTCA-3' and 5'-GTGATTTGATGCCAC GGTAGAA-3'; viii) Cd86: 5'-GGTCGTCTACAAAGCAGTCA AAAA-3' and 5'-AACACCACTGTCCTGCTTGGA-3'. Relative expression levels of these mRNAs were calculated as fold changes versus sham control using the $2^{-\Delta \Delta C T}$ method. Tissue samples were 
A

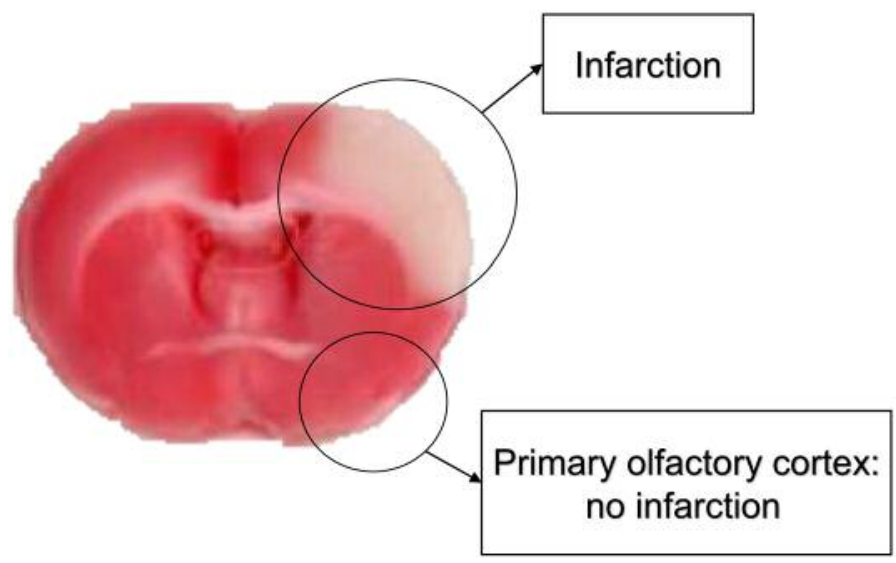

B

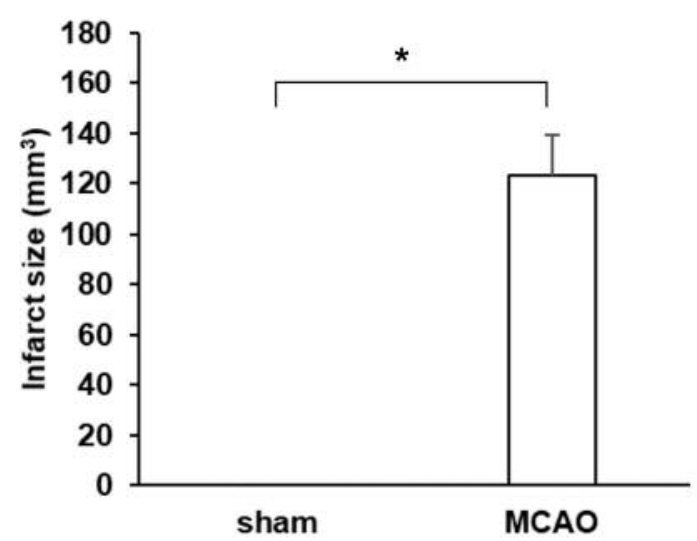

Figure 1. The right middle cerebral artery occlusion (MCAO) produced a significant infarct area on left cerebral cortex in rats. (A) Representative, TTC stained brain slice from rats at 3 days after MCAO. The pale region indicates the infarct area. Right MCAO caused left cerebral cortex infarction; however, the olfactory bulb and primary olfactory cortex were not involved. (B) Quantification of infarct volume of rats at 3 days after $M C A O$ ( $n=4 /$ group). The sham-operated rats showed no infarction. Data are presented as mean $\pm S E M . * p<0.001$.
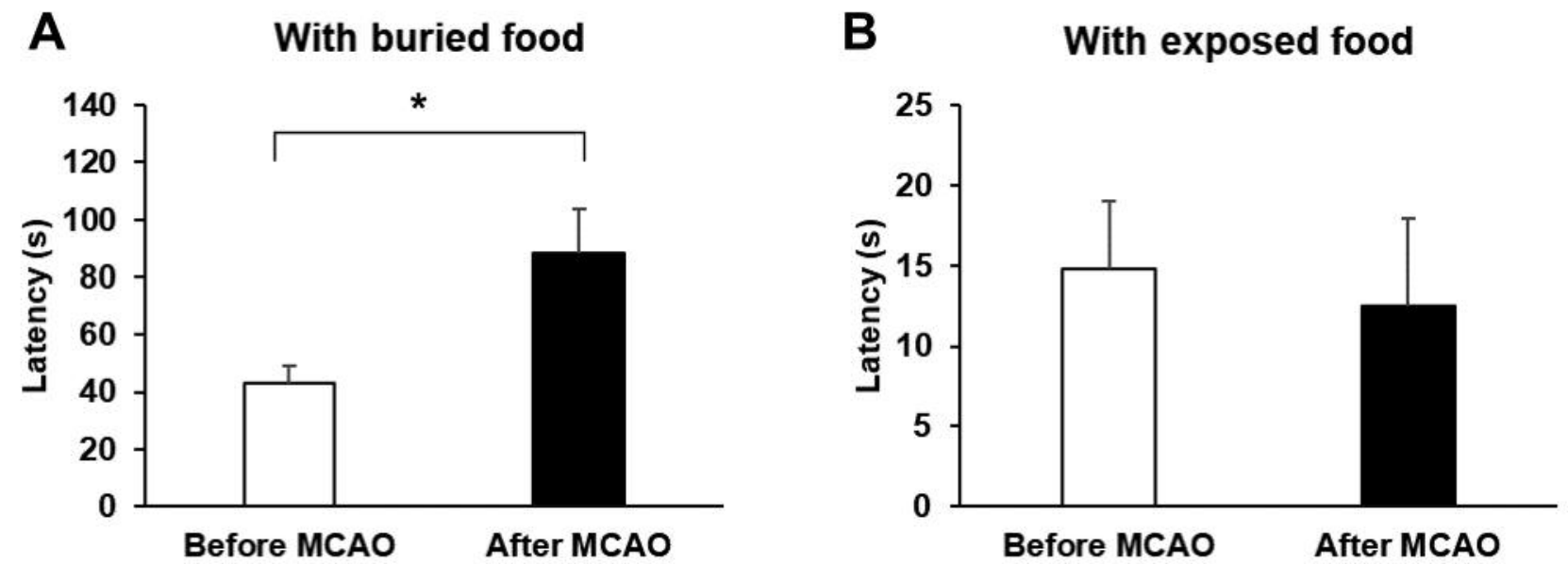

Figure 2. Performance of rats before and after middle cerebral artery occlusion (MCAO) on buried or exposed food tests ( $n=6 /$ group). (A) With buried food, rats after MCAO spent more time to find the food. (B) With exposed food, there was no significant difference in time for finding food between rats before and following MCAO. Data are presented as mean $\pm S E M .^{*} p<0.05$.

normalized to Tbp. Melting curves were routinely performed to assess the specificity of the PCR reaction.

Statistical analysis. Data are expressed as mean \pm standard error of the mean (SEM). Independent-samples $t$-test was used to analyze the difference between two groups. Pearson's correlation was used for correlation analysis between the mRNA expression and the latency of buried food test. All statistical analyses were conducted using the SPSS 24.0 software package (SPSS Inc., Chicago, IL, USA). Results were considered significant at a $p$-value less than 0.05 .

\section{Results}

MCAO produced significant cerebral cortex infarction. TTC stain was performed to evaluate the ischemic region, which showed a pale color. The ischemic region was confined to cerebral cortex, while olfactory bulb as well as primary olfactory cortex were not involved (Figure 1A). At 3 days after MCAO, the mean infarct volume of the rats subjected to MCAO was $123 \mathrm{~mm}^{3}$, and the sham rats revealed no infarction $(p<0.001)$ (Figure 1B). 

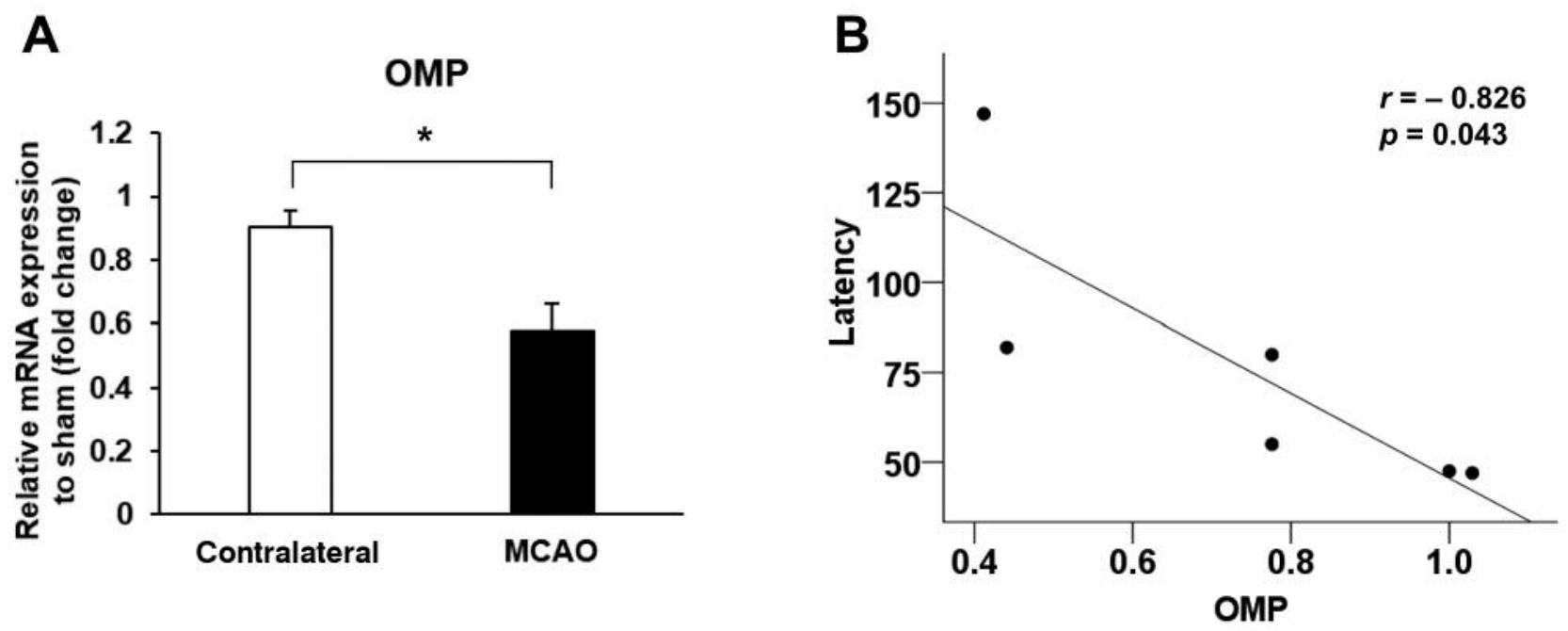

Figure 3. Middle cerebral artery occlusion (MCAO) affected the mRNA expression of the olfactory marker protein (OMP). (A) The mRNA expression of OMP was decreased in the ipsilateral olfactory bulb (MCAO side) after MCAO. (B) The mRNA expression of OMP was negatively correlated with the latency of buried food test. Data are presented as a mean \pm SEM of 4 independent experiments. ${ }^{*}<0.05$.

MCAO induced olfactory dysfunction. We conducted a buried food test to evaluate the olfaction of the rats. The time point of the test was before MCAO and at 3 days after MCAO. On average, rats before MCAO required approximately 43.2 seconds to find the food. In contrast, the average latency for rats subjected to MCAO to find the hidden food was approximately 88.3 seconds $(p=0.022)$ (Figure 2A). For exposed food test, rats before MCAO and after MCAO required 14.8 and 12.6 seconds to reach the food $(p=0.752)$ and there was no significant difference (Figure 2B). During the test, rats after MCAO kept their ability of moving and digging, so the longer latency was mainly caused by olfactory dysfunction instead of strokerelated motor impairment.

MCAO attenuated the OMP expression in the olfactory bulb. OMP is a marker of mature olfactory sensory neuron, and the expression of OMP is positively associated with olfactory function. At 3 days after MCAO, we observed that the mRNA expression of OMP in the ipsilateral olfactory bulb (MCAO side) was decreased, compared to the contralateral side $(p=0.022)$ (Figure 3A). As shown in Figure $3 \mathrm{~B}$, the mRNA expression of OMP was negatively correlated with the latency of buried food test $(r=-0.826$, $p=0.043$ ).

MCAO triggered microglia/macrophage activation in the olfactory bulb. We analyzed the mRNA expression of the markers of microglia/macrophage at 3 days after MCAO. Iba-1 is the marker of microglia/macrophage, and CD68 is a marker of phagocytic cells (15). During microglia/macrophage activation, these can differentiate into M1 microglia/ macrophage, which is a proinflammatory type (8). The markers for M1 microglia/macrophage are CD16 and CD86. At 3 days after MCAO, the mRNA expressions of Iba-1, CD68, CD16 and CD86 were increased in the ipsilateral olfactory bulb (MCAO side), compared to the contralateral side ( $p=0.004$, $0.001,0.022$ and 0.039, respectively) (Figure 4).

MCAO enhanced proinflammatory mediator expression in the olfactory bulb. IL-1 $\beta$ and IL-6 are proinflammatory cytokines, which can enhance the degree of inflammation $(16,17)$. MCP-1 is a proinflammatory mediator and has a role in the recruitment of macrophages (17). At 3 days after MCAO, the mRNA expressions of IL-1 $\beta$ and IL-6 were increased in the ipsilateral olfactory bulb (MCAO side), compared to the contralateral side $(p=0.008$ and $p<0.001$, respectively) (Figure 5). The mRNA expression of MCP-1 increased in the ipsilateral olfactory bulb (MCAO side), compared to the contralateral side $(p=0.023)$ (Figure 5).

\section{Discussion}

Olfactory dysfunction can be induced by ischemic stroke (3, 4). As there are still no studies discussing the pathogenesis between stroke and olfactory function, we thus designed the current study to elucidate this issue, and we first proved that MCAO can induce olfactory dysfunction in rats. The olfactory system consists olfactory receptor, olfactory bulb, olfactory tract, and olfactory cortex. The main blood supply 

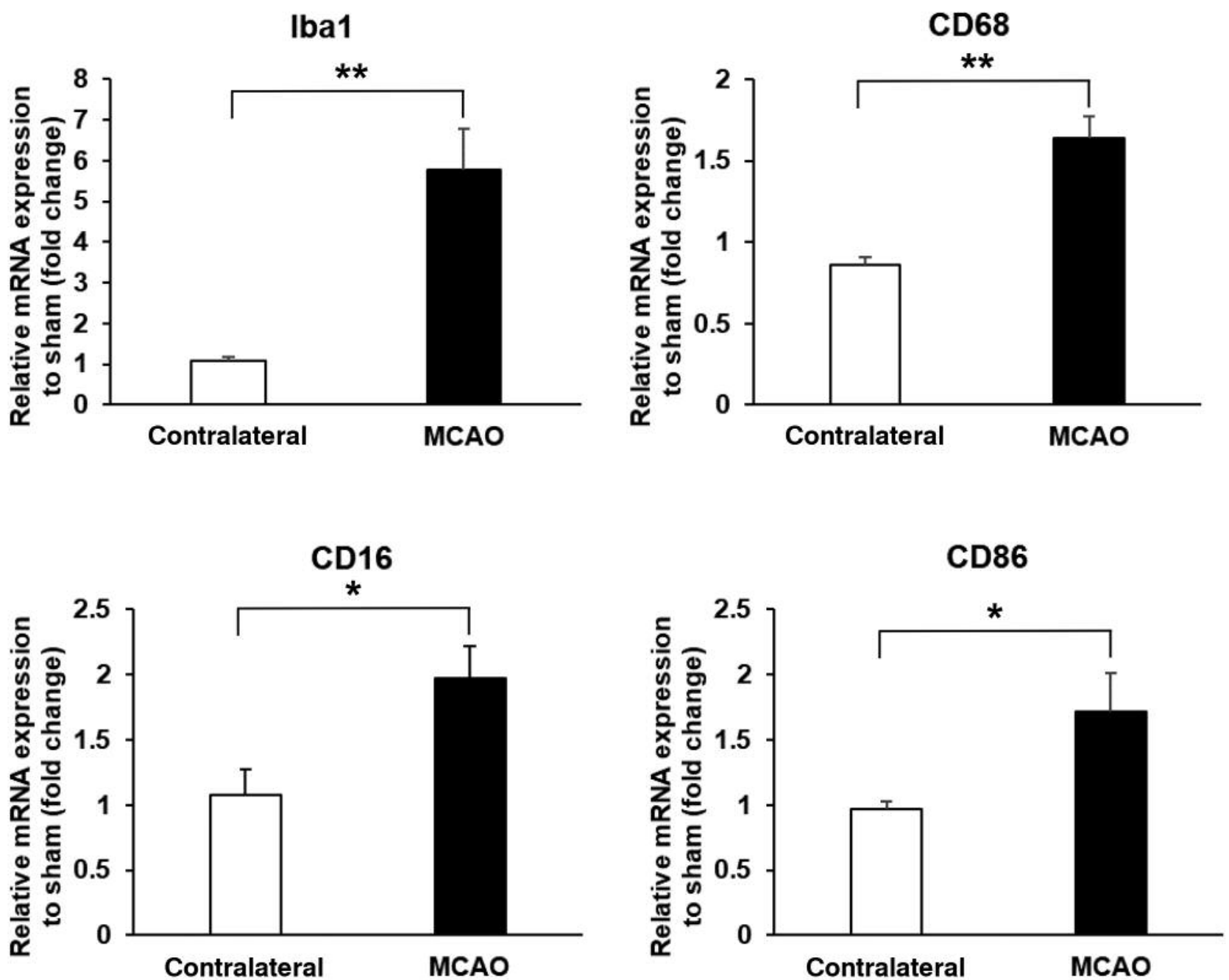

Figure 4. Middle cerebral artery occlusion (MCAO) attenuated the mRNA expression of markers of microglia/macrophage activation (Ibal, $n=4 /$ group), phagocytic cells (CD68, $n=5 /$ group), M1 microglia/macrophage (CD16 and CD86, $n=5 /$ group) in the ipsilateral olfactory bulb (MCAO side). Data are presented as a mean \pm SEM of 4 independent experiments. ${ }^{*} p<0.05,{ }^{* *} p<0.01$.

of the olfactory system is anterior cerebral artery. Thus, MCAO-induced olfactory dysfunction may not be related to primary ischemic injury. In our current study focusing on mRNA expression, we found that microglia/macrophage activation and proinflammatory mediator production in the olfactory bulb may be involved in the pathogenesis of MCAO-induced olfactory dysfunction, which has not been mentioned in previous studies.

Most ischemic strokes arise from the interruption of blood supply of MCA (18). Here we used our newly-developed technique to simulate ischemic cortex injury (13). Because the infarct area did not contain the olfactory system including olfactory cortex, we can study how the MCAO induce olfactory dysfunction separately from the primary ischemic mechanism. Other technique for MCAO, such as intraluminal occlusion is not appropriate for this study because it causes large infarct area, which may involve the olfactory cortex (19).

Buried food test is one of the most commonly used methods for the evaluation of the olfactory function and is able to determine a rodent's olfactory perceptibility (20). To confirm that the buried food test was based on olfaction and not on vision, exposed food test was also arranged. If the motor impairment after MCAO can in general impede the animals to seek the food and subsequently interfere with the olfactory test is another issue. We observed the rats after MCAO and ensured they kept their ability to move and dig, thus the buried food test was still appropriate in our current 

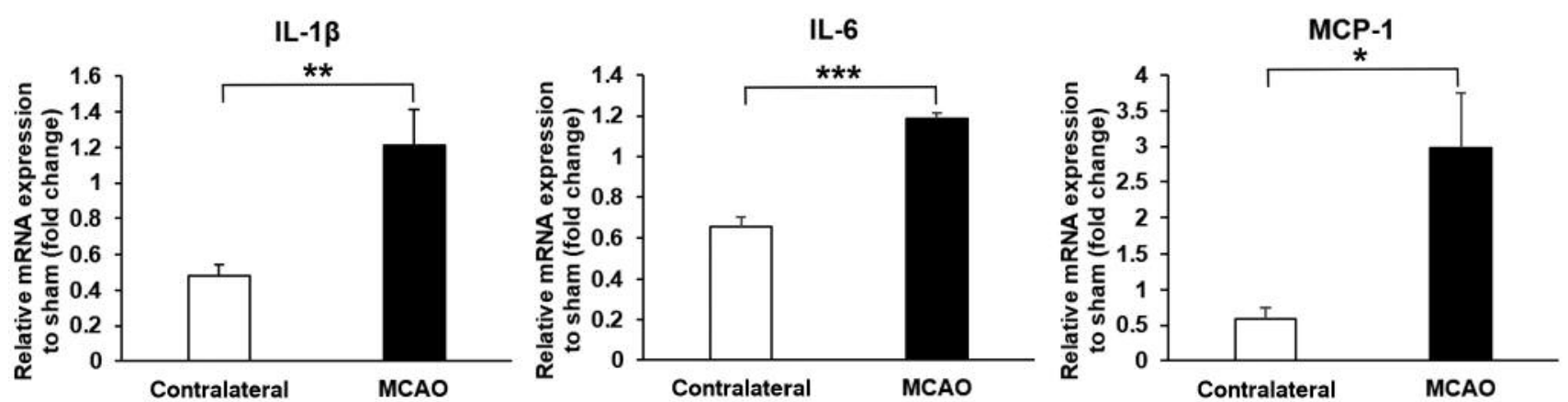

Figure 5. Middle cerebral artery occlusion (MCAO) attenuated the mRNA expressions of proinflammatory mediators (IL-1 $\beta, I L-6$ and MCP-1) in the ipsilateral olfactory bulb (MCAO side) after MCAO (IL-1 $\beta, n=5 /$ group; IL-6 and MCP-1, n=4/group). Data are presented as a mean $\pm S E M$ of 4 independent experiments. $* p<0.05, * * p<0.01, * * * p<0.001$.

MCAO model. Other MCAO models, such as intraluminal occlusion, lead to severe motor impairment, and even rats after MCAO with normal olfaction still cannot approach the buried food. As expected, this is not an ideal model for the current study.

Olfactory dysfunction can be caused by olfactory bulb microglia activation, which can be induced by Alzheimer's, Parkinson's, and Niemann-Pick disease type C $(10,21)$. In a previous study by our group, rats after MCAO had microglia and macrophage activation in the ischemic side of the brain (22). In the current study, we firstly proved that the gene expression of microglia and macrophage markers were upregulated in the olfactory balb after MCAO. After activation, microglia and macrophage can develop into two distinct type: M1 proinflammatory type and M2 anti-inflammatory type (8). M1 type microglia and macrophage can enhance the production of proinflammatory cytokines such as IL- $1 \beta$, IL-6 and TNF- $\alpha$. Instead, M2 type microglia and macrophage can secret anti-inflammatory cytokines (8). Here, we showed that the gene expression of IL-1 $\beta$ and IL- 6 were upregulated in the olfactory bulb after MCAO, indicating a process of neuroinflammation. We also found that the expression of MCP1 , which can promote macrophage recruitment, was also enhanced in the olfactory bulb after MCAO $(17,22)$. Besides, the activation of microglia/macrophage and the production of proinflammatory mediators in the olfactory bulb occurs in the ipsilateral side with respect to the MCAO. The contralateral side remained not influenced, obviously.

In conclusion, this study described a novel pathogenesis of ischemic stroke-induced olfactory dysfunction. Ischemic stroke leads to microglia/macrophage activation and promotes neuroinflammation in the olfactory bulb, causing olfactory dysfunction. Further understanding of the underlying pathogenesis in this pathway can lead to development of drugs that may be used for the treatment of olfactory dysfunction after ischemic stroke in the future.

\section{Conflicts of Interest}

The Authors declare that they have no competing interests in regard to this study.

\section{Authors' Contributions}

Conceptualization was done by CFY, TYC, MYL and YYL; Data curation was done by CFY, TYC, YCC, WHW and YYL; Investigation was done by CFY and YYL; Methodology was done by CFY, TYC and YCC; Supervision was done by YYL; Writing of the original draft was done by CFY; Writing, reviewing and editing were done by CFY, TYC and YYL.

\section{Acknowledgements}

This work was supported by the Ministry of Science and Technology of Taiwan (Grant number 105-2314-B-075-069-MY3, 106-2221-E-010-009, 106-3114-B-010-00, 107-2314-B-075-015MY2, 107-2321-B-039-004, 108-2314-B-075-015 and 108-2643-F008-003), and the Taipei Veterans General Hospital (Grant numbers V106D21-001-MY2-1, V107C-044, and V108C-048 to Yung-Yang Lin; V108B-028 to Chien-Fu Yeh). We also thank for the assistance of experiments by Ching-Han Tsai and Hao-Xiang Huang.

\section{References}

1 Murphy C, Schubert CR, Cruickshanks KJ, Klein BE, Klein R and Nondahl DM: Prevalence of olfactory impairment in older adults. JAMA 288(18): 2307-2312, 2002. PMID: 12425708. DOI: $10.1001 /$ jama.288.18.2307

2 Ciofalo A, Filiaci F, Romeo R, Zambetti G and Vestri AR: Epidemiological aspects of olfactory dysfunction. Rhinology 44(1): 78-82, 2006. PMID: 16550956.

3 Heckmann JG, Stossel C, Lang CJ, Neundorfer B, Tomandl B and Hummel T: Taste disorders in acute stroke: A prospective observational study on taste disorders in 102 stroke patients. Stroke 36(8): 1690-1694, 2005. PMID: 16002758. DOI: 10.1161/01.STR.0000173174.79773.d3 
4 Wehling E, Naess H, Wollschlaeger D, Hofstad H, Bramerson A, Bende $\mathrm{M}$ and Nordin S: Olfactory dysfunction in chronic stroke patients. BMC Neurol 15: 199, 2015. PMID: 26459234. DOI: $10.1186 / \mathrm{s} 12883-015-0463-5$

5 Sacco RL, Wolf PA and Gorelick PB: Risk factors and their management for stroke prevention: Outlook for 1999 and beyond. Neurology 53(7 Suppl 4): S15-24, 1999. PMID: 10532644.

6 Woodruff TM, Thundyil J, Tang SC, Sobey CG, Taylor SM and Arumugam TV: Pathophysiology, treatment, and animal and cellular models of human ischemic stroke. Mol Neurodegener 6(1): 11, 2011. PMID: 21266064. DOI: 10.1186/1750-1326-6-11

7 Jin R, Yang G and Li G: Inflammatory mechanisms in ischemic stroke: Role of inflammatory cells. J Leukoc Biol 87(5): 779789, 2010. PMID: 20130219. DOI: 10.1189/jlb.1109766

$8 \mathrm{Hu}$ X, Leak RK, Shi Y, Suenaga J, Gao Y, Zheng P and Chen J: Microglial and macrophage polarization-new prospects for brain repair. Nat Rev Neurol 11(1): 56-64, 2015. PMID: 25385337. DOI: $10.1038 /$ nrneurol 2014.207

9 Lalancette-Hebert M, Phaneuf D, Soucy G, Weng YC and Kriz $\mathrm{J}$ : Live imaging of toll-like receptor 2 response in cerebral ischaemia reveals a role of olfactory bulb microglia as modulators of inflammation. Brain 132(Pt 4): 940-954, 2009. PMID: 19153151. DOI: 10.1093/brain/awn345

10 Seo Y, Kim HS, Shin Y, Kang I, Choi SW, Yu KR, Seo KW and Kang KS: Excessive microglial activation aggravates olfactory dysfunction by impeding the survival of newborn neurons in the olfactory bulb of niemann-pick disease type c1 mice. Biochim Biophys Acta 1842(11): 2193-2203, 2014. PMID: 25132229. DOI: $10.1016 /$ j.bbadis.2014.08.005

11 Albeanu DF, Provost AC, Agarwal P, Soucy ER, Zak JD and Murthy VN: Olfactory marker protein (omp) regulates formation and refinement of the olfactory glomerular map. Nat Commun 9(1): 5073, 2018. PMID: 30498219. DOI: 10.1038/s41467-01807544-9

12 Hasegawa-Ishii S, Shimada A and Imamura F: Lipopolysaccharide-initiated persistent rhinitis causes gliosis and synaptic loss in the olfactory bulb. Sci Rep 7(1): 11605, 2017. PMID: 28912588. DOI: 10.1038/s41598-017-10229-w

13 Yeh CF, Chuang TY, Hung YW, Lan MY, Tsai CH, Huang HX and Lin YY: Development of a modified surgical technique for simulating ischemic cerebral cortex injury in rats. In Vivo 33(4): 1175-1181, 2019. PMID: 31280207. DOI: 10.21873/invivo. 11588

14 Alberts JR and Galef BG Jr.: Acute anosmia in the rat: A behavioral test of a peripherally-induced olfactory deficit. Physiol Behav 6(5): 619-621, 1971. DOI: 10.1016/00319384(71)90218-6
15 Hopperton KE, Mohammad D, Trépanier MO, Giuliano V and Bazinet RP: Markers of microglia in post-mortem brain samples from patients with Alzheimer's disease: a systematic review. Mol Psychiatry 23(2): 177-198, 2018. PMID: 29230021. DOI: 10.1038/mp.2017.246

16 Shaftel SS, Griffin WS and O'Banion MK: The role of interleukin-1 in neuroinflammation and Alzheimer disease: an evolving perspective. J Neuroinflammation 5: 7, 2008. PMID: 18302763. DOI: $10.1186 / 1742-2094-5-7$

17 Scheller J, Chalaris A, Schmidt-Arras D and Rose-John S: The pro- and anti-inflammatory properties of the cytokine interleukin-6. Biochim Biophys Acta 1813(5): 878-88, 2011. PMID: 21296109. DOI: 10.1016/j.bbamcr.2011.01.034

18 Jang SH and Chang MC: Motor outcomes of patients with a complete middle cerebral artery territory infarct. Neural Regen Res 8(20): 1892-1897, 2013. PMID: 25206498. DOI: 10.3969/ j.issn.1673-5374.2013.20.008

19 Fluri F, Schuhmann MK and Kleinschnitz C: Animal models of ischemic stroke and their application in clinical research. Drug Des Devel Ther 9: 3445-3454, 2015. PMID: 26170628. DOI: 10.2147/DDDT.S56071

20 Seo Y, Kim HS and Kang KS: Microglial involvement in the development of olfactory dysfunction. J Vet Sci 19(3): 319-330, 2018. PMID: 29032655. DOI: 10.4142/jvs.2018.19.3.319

21 Kohl Z, Schlachetzki JC, Feldewerth J, Hornauer P, Munch M, Adame A, Riemenschneider MJ, Winkler $\mathrm{J}$ and Masliah E: Distinct pattern of microgliosis in the olfactory bulb of neurodegenerative proteinopathies. Neural Plast 2017: 3851262, 2017. PMID: 28409032. DOI: $10.1155 / 2017 / 3851262$

22 Yeh CF, Chuang TY, Hung YW, Lan MY, Tsai CH, Huang HX and Lin YY: Inhibition of soluble epoxide hydrolase regulates monocyte/macrophage polarization and improves neurological outcome in a rat model of ischemic stroke. Neuroreport 30(8): 567-572, 2019. PMID: 30950936. DOI: 10.1097/WNR.0000 000000001248
Received July 18, 2019

Revised August 5, 2019

Accepted August 7, 2019 